\title{
A Review of Mechanical Properties and Durability of Nano-concrete
}

\author{
Wei Li ${ }^{1,2,3}$ a , Linkai Xiao ${ }^{1, b}$, Xiaochu Wang ${ }^{1,2,3 c}$ and Yi Ran ${ }^{1, d}$ \\ ${ }^{1}$ Shenyang University, China \\ 2 of Resources \& Civil Engineering, Northeastern University, China \\ ${ }^{3}$ Liaoning Provincial Key Laboratory of Geoenvironmental Engineering, China

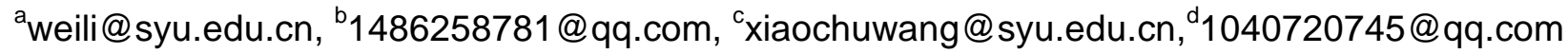

Keywords: Nano-concrete; Mechanical properties; Durability; Optimum dosage.

Abstract: The incorporation of nano-materials in concrete, its various aspects of the performance have been improved, but also need to further understand the nano-materials to improve the performance of concrete deficiencies. Due to its excellent mechanical properties and durability, nano-concrete is becoming more and more popular in practical engineering. In order to get a deep understanding of the mechanical properties and durability of nano-concrete, as well as the optimal amount of nano-materials, scientists have done a lot of research in this area. In this paper, the most commonly is used concrete mechanical properties and durability of the two aspects of research, and analysis of the future development trend of nano-concrete.

\section{Introduction}

Concrete is the main material of modern architecture. With the increase of population and the development of industrialization, the amount of concrete is also increasing. Therefore, concrete is one of the important raw materials for industrial construction. As an important industrial material, concrete has many advantages, such as easy molding, good compression resistance, high stiffness and wide range of raw materials, but it can not be applied in some aspects.

Nanometer material ${ }^{[1]}$ refers to the ultrafine material with particle size at the nanometer level $(1-100 \mathrm{~nm})$, which is larger than the atomic cluster (the cluster of atoms less than $1 \mathrm{~nm}$ in size) and smaller than the ordinary powder, in the transition zone of the cluster to the macroscopic object,A typical mesoscopic system.Because of its small size, large surface area and other factors, have the following four special effects: size effect, quantum effect, surface effect and interface effect. These four effects mainly reflect the structural, chemical and physical characteristics of nano-materials, which are called "the most promising materials of the 21st century".Nano-mineral powder mainly includes nano-silica, nano-calcium carbonate and nano-silica powder. Ordinary Portland cement within the distribution of 10-100nm gel pores, the incorporation of nano-powder just fill these gaps, play a role in improving the permeability of concrete and toughness.

\section{Study on Mechanical Properties of Nanometer Concrete}

The results show that the nano-materials are incorporated into the concrete, which can effectively improve the mechanical properties such as compressive and flexural properties of concrete. The incorporation of nano-silica improves the mechanical properties of concrete much higher than that of nano-calcium carbonate.

The reason is that the volcanic ash activity of silica is much higher than that of silica fume, and the incorporation of silica makes the slurry fluidity decrease and the aggregation time is greatly shortened. At the same time, the incorporation of nano-silica can also greatly improve the concrete the early strength.Nano-silica puts into the cement, due to its structure, physical and chemical characteristics, and cement in a large number of $\mathrm{Ca}(\mathrm{OH})_{2}$ combined, thereby promoting cement hydration, improve the chemical reaction of the exothermic efficiency, so that the cement slurry Micro-structure to improve the cement more uniform and dense, as shown in Figure 1,2.Nano-calcium carbonate ${ }^{[2]}$ incorporation, play a physical and chemical effects, reduce the internal surface area of cement stone to 
speed up the early hydration of clinker speed, reduce porosity, increase the density of cement stone, and thus improve the compressive strength of cement 3,4.

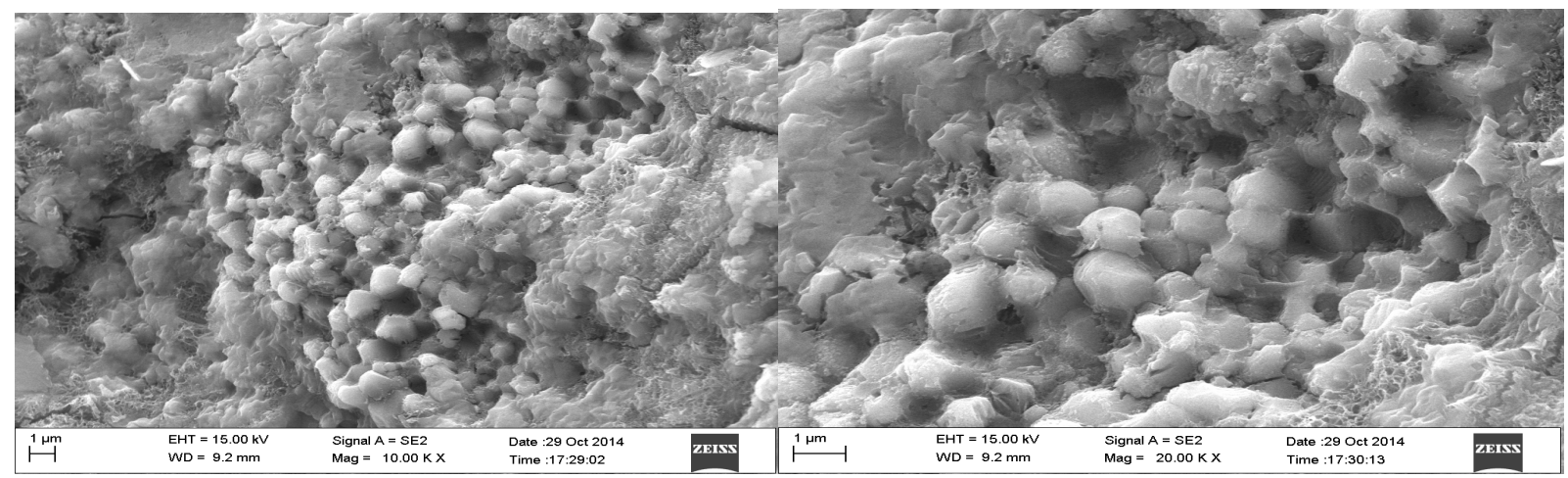

Fig. 1, 10K times NS20C scanning electron microscope Fig. 2,20K times NS20C scanning electron microscop

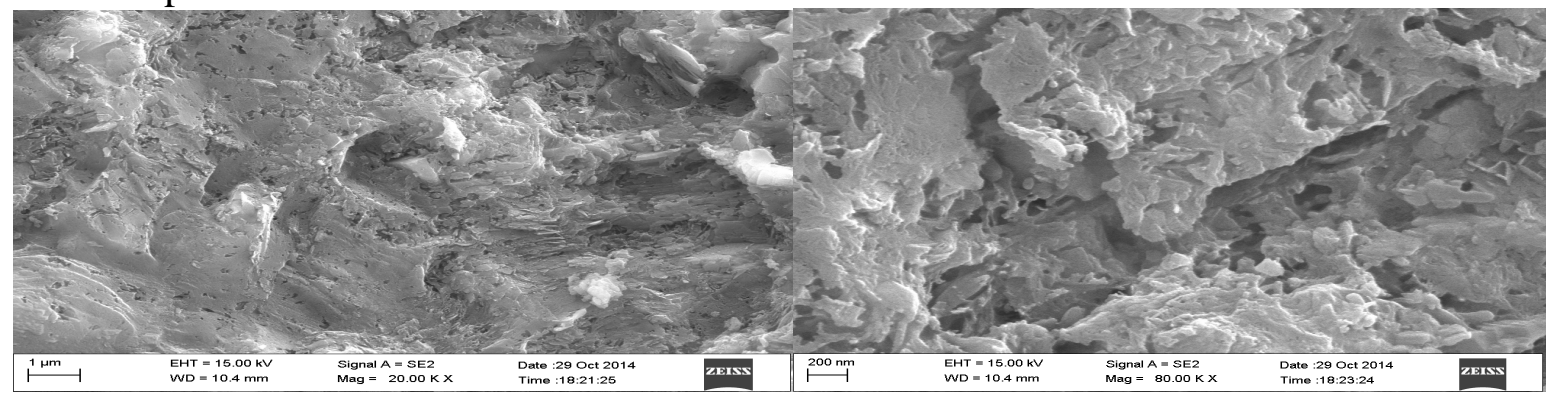

Fig. 3, 20K times NC05C scanning electron microscop Fig. 4, 80K times NC05C scanning electron microscop

Fangliang $\mathrm{Cao}^{[3]}$ and so on for nano-materials to do a comparative test, the experiment will be divided into three groups: not joined the nano-materials of concrete, adding nano-silica concrete and adding nano-calcium carbonate concrete. Through the experimental results shown analysis, the addition of nano-materials to improve the compressive strength of concrete. The incorporation of nano-silica increased the compressive strength of concrete by $4 \%$. The concrete was mixed with different nano-materials for 28 days. It was found that the compressive strength of concrete doped with nano-calcium carbonate was higher than that of the nano- Strength increased by $16.7 \%$, while also analysis of nano-silica and nano-calcium carbonate $e^{[4]}$, the best dosage of $0.5 \%$ and $3 \%$. The experimental results also show that nanomaterials can reduce the fluidity of concre.

. Li Guhua ${ }^{[5][6][7]}$ and other on the performance of nano-concrete system of experimental study, they believe that the incorporation of nano-materials can improve the early strength of concrete, the most typical is the strength of concrete in the 7 days when the most significant, then mixed with 5\% Silica is more pronounced than the incorporation of 3\% nano-silica, which is not related to the amount of nano-silica, although it is not obvious or even better than the addition of nanomaterials. When the amount of nano-silica is $5 \%$, the strength of the concrete for 60 days is not as good as that of the nano-materials. However, the incorporation of nano-silica into $3 \%$ has obvious effect on improving the late strength of concrete.

TANG Xiao-ping, WEI Xiu-ying ${ }^{[8]}$ et al. A similar experimental study was carried out using nanosilica and dichloroxichloride. The results were compared with the incorporation of three different nanomaterials. The results showed that the incorporation of the nanocomposites was corresponding to the concrete $3 \mathrm{~d}, 7 \mathrm{~d}, 28 \mathrm{~d}$ Compressive strength is $20 \%, 15 \%, 10 \%$.

\section{Study of Nano - concrete Impermeability}

Nano-silica with anti-cracking, impermeability, frost and so on.It is found that nano-silica can improve the internal structure and comprehensive properties of concrete, fill the internal voids of 
concrete, increase its density, improve the crack resistance, anti-freeze and impermeability of concrete and improve the durability of concrete.

$\mathrm{Han} \mathrm{Li}$ and so on with sodium chloride solution soak the standard sample $24 \mathrm{~h}$, and then remove the drying test method, cycle 10 times. The chloride ion content of the concrete was $92.7 \%, 92.3 \%$ and $91.9 \%$ of the mixed concrete at $0.5 \%, 1 \%$ and $2.0 \%$, respectively, when the nano-silica at $2.5 \mathrm{~m}$ depth was measured.Chengming Lan also made a similar anti-chloride ion test, obtained a very similar experimental results.

\section{Nano Concrete Frost Resistance}

Frost resistance is one of the keys to the durability of concrete, and one of the factors that affect the durability of concrete is the cycle of freezing and thawing.Because the strength of concrete is directly related to the safety and performance of building structure, so its mechanical properties of concern.In order to ensure the durability of the building structure in the use of the process, frost resistance in real life is significant.

According to the freezing and thawing M7.5 specimens, the internal structure of the concrete after adding nanometer material was greatly improved, and the internal structure of the concrete was improved greatly. The pore size of $150 \mathrm{~mm}$ disappears from the inside of concrete, greatly increases the internal density of concrete, and avoids structural damage caused by hydrostatic theory and osmotic pressure theory.Xiaolin Ren and Shunkai Li studied the same similar comparison test. It was found that under the condition of -10C, 25 times freeze-thawing was carried out, the incorporation of nanometer material $0.75 \%$ concrete strength loss was $3.2 \%$, and the reference concrete loss rate was $8.6 \%$, so the nanometer material In the concrete, the frost resistance of concrete is obviously improved.

The results show that the east elastic modulus of concrete decreases with the increase of nano-silica content, and is mixed with $0,1 \%, 3 \%$ and $5 \%$. The flexural modulus loss of silica concrete was $15.3 \%$, $14.9 \%, 7.0 \%$ and $3.3 \%$ respectively.

\section{Conclusions}

The above has been shown by compressive strength, flexural strength test, shrinkage test, anti-carbonization test, resistance to chloride ion penetration test and freeze-thaw test.In this paper, through the microscopic research techniques such as scanning electron microscopy and phase analysis, it is found that nanomaterials are helpful to optimize the internal pores and microcracks of concrete, enhance the compactness of concrete.In addition, nano- $\mathrm{SiO}_{2}$ with volcanic ash activity can reduce the formation of $\mathrm{Ca}(\mathrm{OH})_{2}$ and reduce the orientation of concrete, and improve the transition zone strength of cement-based materials and aggregates.Comparing the mechanical properties and durability of nano-concrete under different dosage.The effects of nanomaterials on the properties of nano-composites were studied by combining the scanning electron microscopy and phase analysis.

The above has shown that the incorporation of nanomaterials ${ }^{[9]}$, especially nano-silica and nano-calcium carbonate, has greatly improved the mechanical properties and durability of concrete ${ }^{[10]}$. However, the two commonly used nanomaterials are expensive to bring economic pressure to construction cost,this limits their practical use in concrete and requires a more economical and affordable nanomaterial to be incorporated into the concrete. And then there is a small nano-particles, the specific surface area and other characteristics, making it difficult to disperse in the cement, on the contrary is also easy to unite, leading to the compressive strength of concrete is not easy to improve.Therefore, how to change the degree of dispersion of nano-materials in concrete, to improve the compressive strength of concrete is the need to continue to study the problem.In addition, it is also very significant to study the special properties of nano-materials into special concrete research, such as ultra-high strength concrete, self-repairing concrete, corrosion-resistant concrete, shock-absorbing concrete, green concrete and intelligent concrete. 


\section{Acknowledgement}

This research was financially supported by the Liaoning province key laboratory of environmental geotechnical engineering project; The supporting project of national science and technology (BAJ15B03 2013-5).

\section{References:}

[1] Mei Zhang. The research status and development of nanometer materials [J]. Journal of missile and space launch technology. 2000 (3).

[2] Tao Meng , kuangliang Qian, xiaoqian, shulin zhan. Nanometer calcium carbonate particles of cement hydration performance and the influence of interfacial properties [J]. Rare metal materials and engineering. 2008 (S2).

[3] Zhengyu Huang, Fangliang Cao. Nano material effect on the properties of high performance concrete, material review [J], 2012.

[4] Zhengyu Huang, Tianyu $\mathrm{Zu}$.Study on the Effect of Nano- $\mathrm{CaCO}_{3}$ on the Properties of Ultra-High Performance Concrete [J] Silicate Bulletin, 2013,32 (6): 1103-1109.

[5] Guhua Li , Bo Gao.Effects of nano-powder $\mathrm{SiO}_{2}$ and $\mathrm{CaCO}_{3}$ on concrete performance [J] Journal of the China Railway Society, 2006,28 (1): 131-136

[6] Guhua Li, Bo Gao.Effects of nano $\mathrm{CaCO}_{3}$ on dry and dry corrosion resistance of concrete [J] Journal of Chongqing Jiaotong University, 2007,26 (2): 131-135.

[7] Guhua Li.Effects of nanomaterials on the durability of concrete [D] Chengdu: Southwest Jiaotong University, 2006.

[8] Xiaoping tang, Xiuying Wei, xiaowen liu, xueyan wang. Nanometer $\mathrm{SiO}_{2}$ for different age concrete mechanics performance test [J]. J tech review. 2011 (21).

[9] Mei Zhang, Huanchun Chen, XujieYang, Lude Lu, Xin Wang.Studies and Prospects of Nanomaterials [J]. submitted to Journal of Materials Research .2003.

[10] Fangliang Cao. The influence of the nano materials of high performance concrete strength research [D]. Hunan university. 2012. 